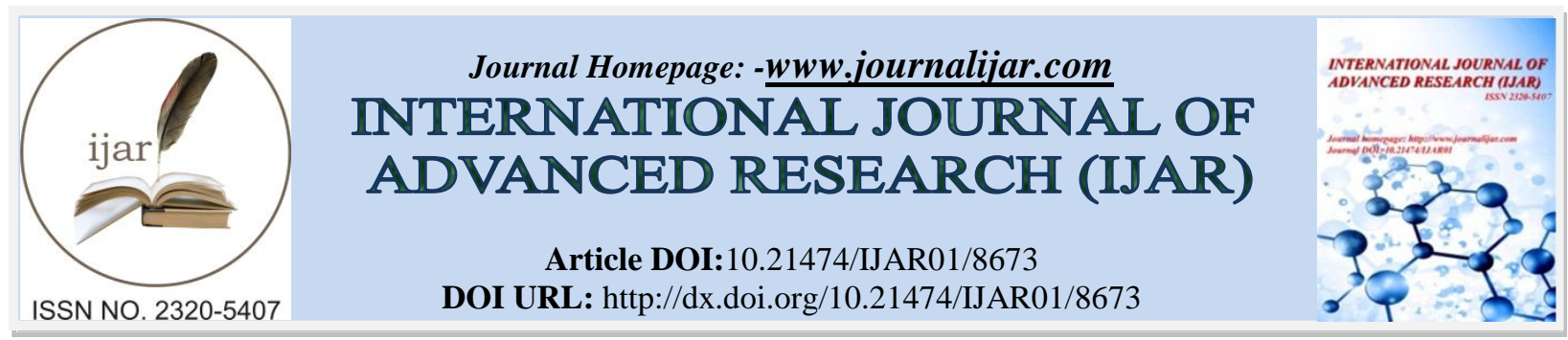

RESEARCH ARTICLE

\title{
SILENT GIANT LEFT ATRIUMA CASE REPORT.
}

\section{Y. Kettani, R. Nhiri, H.kharbouch, S. Hallab, J. Zarzur and M.Cherti.}

Cardiology B service, Ibn Sina Hospital, Rabat-Morocco.

\section{Manuscript Info}

Manuscript History

Received: 12 January 2019

Final Accepted: 14 February 2019

Published: March 2019

Key words:-

Behaviour, determinant, factor, health, seeking.

\begin{abstract}
Left atrial enlargement (LAE) is frequently present in a variety of heart diseases including obviously that secondary to rheumatic fever. At times, the left atrium might become enlarged to giant proportions.

It may, however, be seen in pure or predominant mitral stenosis. We recently saw a forty-five-year-old man with silent progressive huge left atrium with atrial fibrillation whose first clinical manifestation was dyspnea. Aside from mild progressive shortness of breath during the past year, the patient had been asymptomatic all her life. He was presented with cardiomegaly evident on her chest roentgenogram. The cardiac enlargement was due to a giant left atrium that distorted the cardiac structures. An echocardiogram was able to delineate the huge left atrium.
\end{abstract}

Copy Right, IJAR, 2019,. All rights reserved.

\section{Introduction:-}

Left atrial enlargement (LAE) is frequently present in a variety of heart diseases including obviously that secondary to rheumatic fever.(1-4) At times, the left atrium might become enlarged to giant proportions.(5)

It may, however, be seen in pure or predominant mitral stenosis. We recently saw a forty-five-year-old man with silent progressive huge left atrium with atrial fibrillation whose first clinical manifestation was dyspnea.

\section{Case Report}

A 45-year-old man was admitted to Ibn Sina Hospital with a chief complaint of progressive shortness of breath. There was no orthopnea and no paroxysmal nocturnal dyspnea. There was no chest pain or any other cardiac complaint. There were no complaints of voice hoarseness, or any other gastrointestinal symptoms.

The patient stated that he had rheumatic fever as a child and he had also been told that he had a heart murmur. He had, however, been in good health until the increasing dyspnea markedly limited his activities.

The physical examination revealed a blood pressure of $120 / 70 \mathrm{~mm} \mathrm{Hg}$ with an irregular heart rate at 80beats/minute. The neck veins were not distended. The lungs were clear to percussion and auscultation.

The examination of the heart revealed a grade IV/VI middiastolic rumble at the apex.

The abdominal examination yielded normal findings, and there was no peripheral edema.

The electrocardiogram showed atrial fibrillation with a frontal plane axis of $90^{\circ}$. This was compatible with right ventricular enlargement. The chest roentgenogram showed massive cardiomegaly. The chambers that caused this enlargement could not be clearly ascertained (Fig.1) 


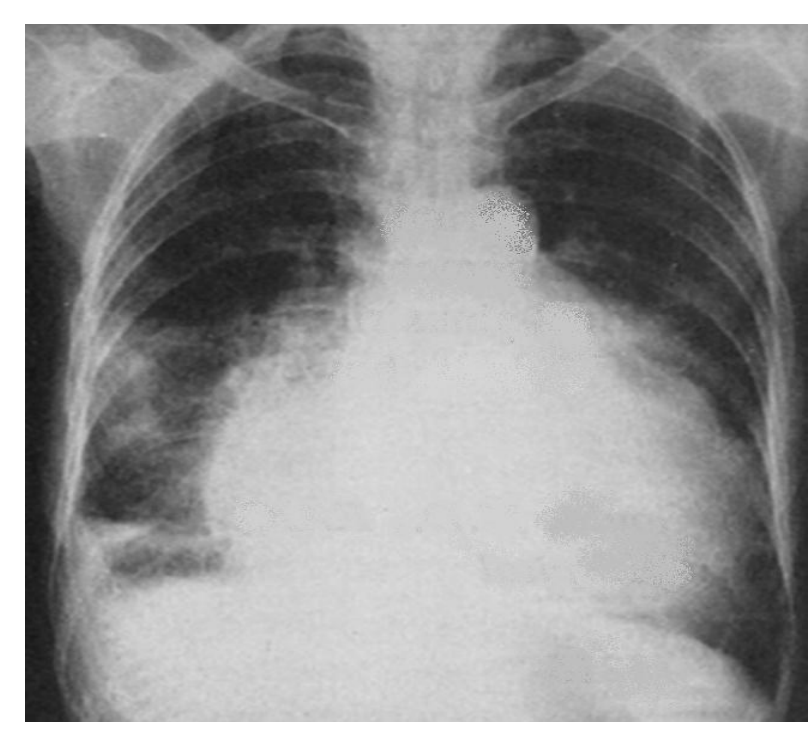

Figure1:-The chest X-ray AP view showed increased cardiothoracic ratio; the left atrial shadow extendedTowards the left and right cardiac borders, with bilateral lung haziness.

Echocardiography (Fig. 2) showed a hugely dilated left atrium, with an anteroposterior diameter of $17 \mathrm{~cm}$ in the parasternal long axis view. The estimated left atrial volume was $2000 \mathrm{ml}$. There was severe mitral stenosis MVA = $0.6 \mathrm{~cm} 2$ by pressure half time and a mean diastolic pressure gradient of $14 \mathrm{mmHg}$ at rest (Fig.3).

Color Doppler showed minimal mitral regurgitation. The left ventricle was enlarged with a systolic ejection fraction of 55\%. The systolic pulmonary artery pressure was $48 \mathrm{mmHg}$.

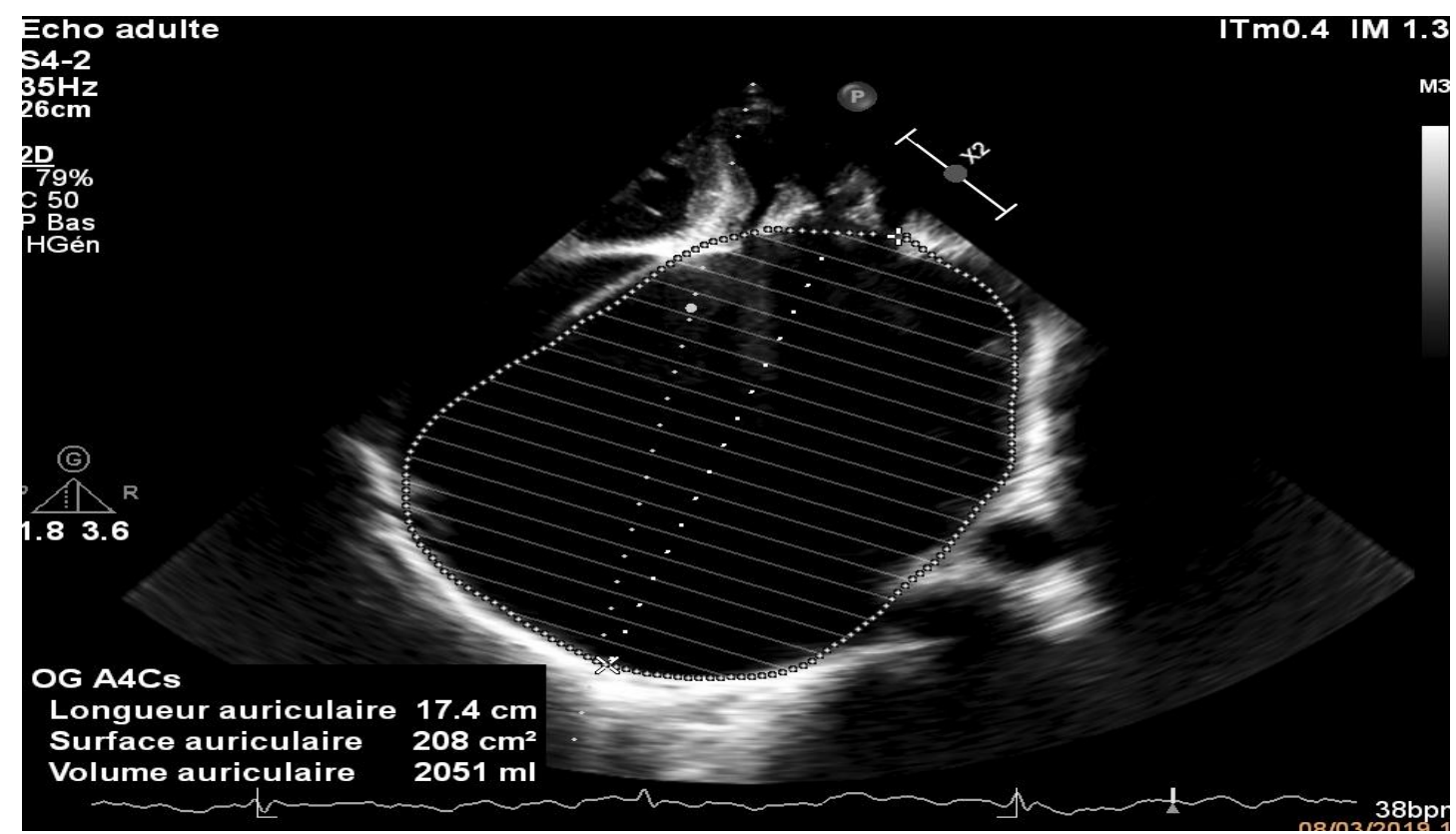

Figure 2:-hugely dilated left atrium 


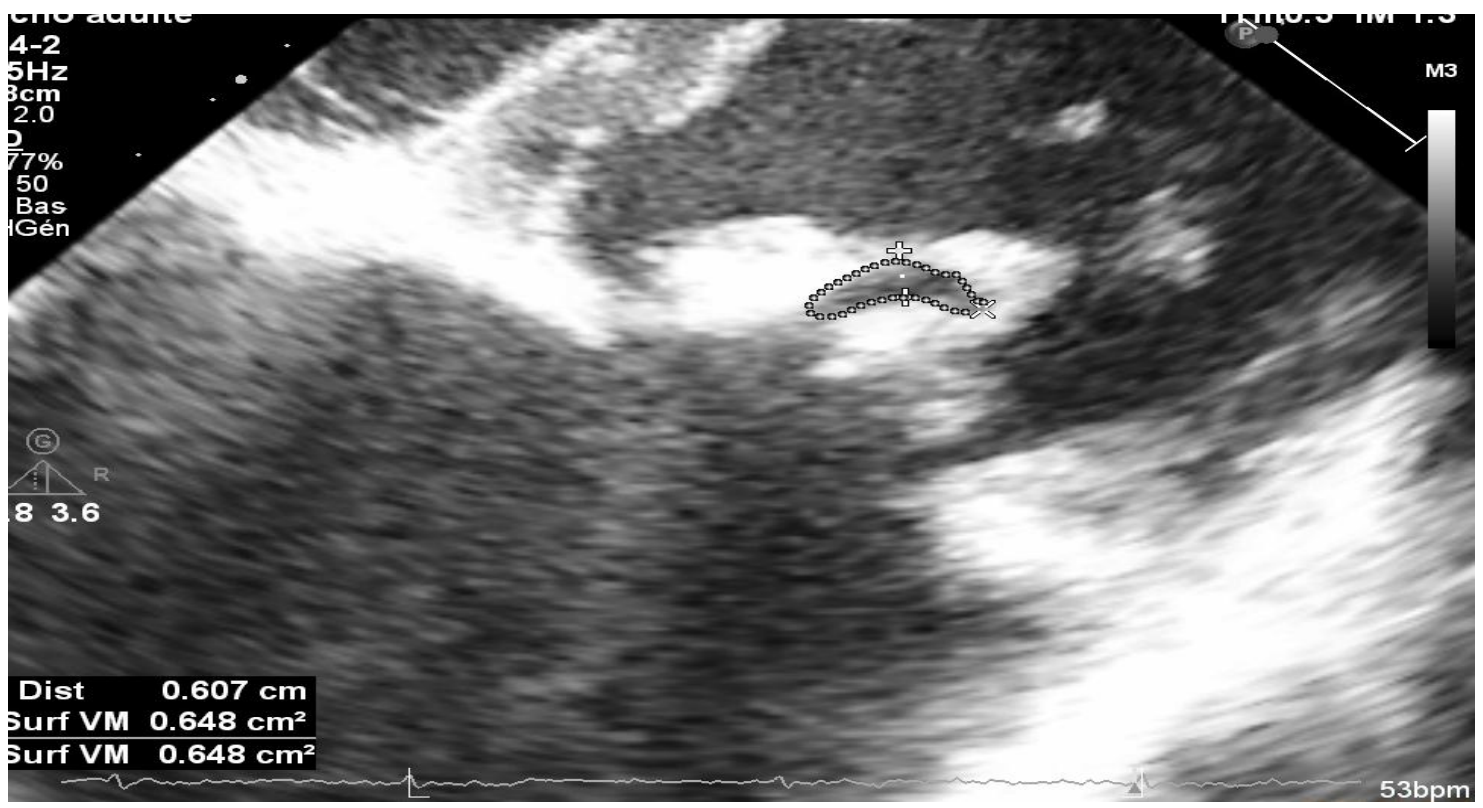

Figure 3:-severe mitral stenosis

A transesophageal study was performed to assess the mitral valve. The giant left atrium was filled with "heavy smoke" shadow but no definite thrombi. The left atrium appendage looked plicated.

A diagnosis of decompensate heart failure syndrome was made, and oral treatment with furosemide, spironolactone and angiotensin receptor blocker was prescribed and warfarin was continued.

The patient was referred to the cardiovascular surgeon for mitral valve replacement and left atrial reduction.

\section{Discussion:-}

Rheumatic heart disease (RHD) continues to be a common health problem in the developing world, causing morbidity and mortality among both children and adults.

Giant left atrium should be kept in mind whenever a patient presents with rheumatic mitral disease history and right lung opacification on chest X-ray (CXR). Hurst (6) defined a giant left atrium as "one that touches the right lateral side of the chest wall" (on CXR) and that "the condition is almost always caused by rheumatic mitral valve disease". He considers the CXR diagnostic of a giant left atrium, when the left atrium touches the right lateral side of the chest wall. He states that no other diagnostic tests are needed because no other condition produces such an unusual abnormality. In his article, Hurst discusses the anatomical location of the normal left atrium: "The normal left atrium is not located on the left; it is located in the middle of the chest; it is the most posterior chamber of the heart, and it abuts the spine and esophagus. When the left atrium enlarges, it moves rightward and will, in the giant left atrium syndrome, touch the right lateral wall of the chest. (6) Piccoli et al (7) defined the giant left atrium as a cardiothoracic ratio on CXR of $>0.7$ combined with a left atrial anterior-posterior diameter of $>8 \mathrm{~cm}$ on transthoracic echocardiography. Other investigators (8) have decreased the LA size for diagnosis to $6.5 \mathrm{~cm}$ in the parasternal long axis view. The lateral extension of the cardiac border reflects left atrial dilatation toward the right borders of the chest wall as seen on the CXR. The echocardiography diameter measures the anteroposterior extension of the left atrium. However, measurement of the left atrial volume will theoretically incorporate the dilatation in both directions. Therefore, we believe that the left atrial volume and left atrial volume index should be incorporated in the definition but this remains to be validated in further studies. There have been two instances in the history of medicine where a giant left atrium was misinterpreted as representing another cardiac pathology and was punctured: in 1901, Owen et al misinterpreted a GLA as pericardial effusion. (8) One hundred years later, Schvartman et al performed a CT-guided biopsy on a GLA believing it to be a malignant mass. (9) Many case reports associate giant left atrium to rheumatic pathology, (10-21) but a few reports associate GLA with a non-rheumatic etiology. Phua et al and Brownsberger et al $(22,23)$ reported GLA due to mitral valve prolapse. Yuksel et al reported it with 
hypertrophic cardiomyopathy. (24) Another case report was published by Cheng et al (25) associating this condition with cardiac amyloidosis, causing a restrictive cardiomyopathy pattern.

Most reports in the literature, as our case presented had a rheumatic origin, as suggested by the history. Hurst (6) attributed the dilatation of the left atrium to the rheumatic process causing pancarditis. However, pathological studies have failed to reveal the Aschoff nodules (fibrinoid necrotic center found in the myocardium surrounding blood vessels, and other regions of the body) in the atrial tissues; rather, these studies found fibrosis with chronic inflammatory findings.(25)

This supports the explanation that the left atrium progressively enlarges secondary to longstanding chronic volume and pressure overload more than being a part of the rheumatic process. Atrial fibrillation, which can occur secondary to left atrial enlargement, contributes more to the increase in overload on the left atrium and causes more enlargements. As mentioned by Hurst (6) and Le Roux, (26) anatomically, the left atrium is not a leftsided structure and this chamber is the most posterior structure of the heart. The four pulmonary veins enter the left atrium in a square shape; the side of which is maximally $4 \mathrm{~cm}$. The left atrium extends more to the left side forming the left atrial appendage. On the right side, the left atrial wall rarely exceeds $1 \mathrm{~cm}$ in width. Anteriorly, the inter-atrial septum separates the left and right atriums. Posteriorly, the left atrium is closely related to the main bronchus of both lungs. The esophagus and the descending thoracic aorta lie posterior to the left atrium. Therefore, giant left atrium can cause intracardiac or extra-cardiac compression manifestations. Minagoe et al (26) studied the IVC flow in giant left atrium. They found a significant reduction in IVC orifice with significant increase in flow velocities, explained by the inter-atrial septal bulge occurring in this condition, which partially obstructs IVC flow. This explains the rightsided manifestations that can occur in those patients even without significant tricuspid regurgitation. (27) Other investigators (28) have reported left lung collapse caused by compression of the distal main bronchus and collapse of the right middle lobe due to direct compression. Posterior displacement of the esophagus is a logical result of left atrial enlargement which can be symptomatic causing dysphagia[( 29) in extreme enlargement of the left atrium. Nigri (30) reported a rare complication of the giant left atrium, pushing the heart to the right side of the thorax mimicking dextrocardia.

Thromboembolism is a major complication of giant left atrium. Many cases have been reported in the literature. Before the widespread use of echocardiography, thrombosed giant left atrium was mistaken for mediastinal tumor. (11) Echocardiography enabled the possible association of left atrial thrombus with giant left atrium. This complication was seen in patients before and after mitral valve replacement.(31,32) Kutay et al (35) compared the incidence of thromboembolic event in patients with and without giant left atrium after mitral valve replacement without left atrial plication. The incidence was comparable in both groups and this was correlated to poor socioeconomic status causing noncompliance to anticoagulation. Anticoagulant therapy can effectively prevent thromboembolic complications with giant left atrium.

Non-compliance to anticoagulation is the major cause for this complication whatever the left atrial size. Therefore, a giant left atrium is an indication for the initiation of anti-coagulant therapy. Our case was anticoagulated with an international normalized ratio range between 2.5 and 3.5. He showed spontaneous echo contrast in the left atrium but no evidence of thrombi.

\section{Role of Surgery:-}

The aim of surgery in giant left atrium is to correct the mitral valve abnormalities, to treat compression manifestations, to prevent thromboembolism and to revert atrial fibrillation to normal sinus rhythm. (33) Two strategies had been applied in the management of mitral valve surgery with giant left atrium. The first is performing mitral valve surgery with left atrial volume reduction while the second is to perform mitral valve surgery alone. (33)

The main indication for volume reduction is the presence of intracardiac or extracardiac compressive symptoms. But some surgeons believe that successful mitral valve surgery alone will result in the eventual reduction of left atrial size as the volume and mean atrial pressure decline. (33)

Surgeons who performed left atrial placation believed that the effect of the rheumatic process on the elastic fibers of the tissue is irreversible. This process causes strain and loss of tone; thus, the left atrial enlargement is irreversible. They claimed that by reducing the left atrial size with replacement of the mitral valve, the pressure effect is reduced with favorable effect on the postoperative course. 
A second indication is the presence of thrombus and a history of thromboembolic events. Left atrial volume reduction can, in theory, prevent recurrent thrombosis by reducing intra-atrial stasis. However, these can be difficult to perceive because such patients are on warfarin from the beginning. Furthermore, a large atrial size increases thromboembolic risk and reduces the success rate of cardioversion. Tamura et al (34) presented a case of severe mitral stenosis and regurgitation. This patient had mitral valve replacement with left atrial volume reduction. The immediate postoperative period showed relief of the compression with widening of the main bronchus diameter and decrease of the angle of the carina with improvement in respiratory functions. Surgeons who treated mitral valve disease with giant left atrium by only replacing the mitral valve believed that prolongation of the cardiopulmonary bypass time in these patients does not warrant left atrial plication except when the atrium compresses the lung as to produce atelectasis. In the study by Johnson and colleagues, (35) left atrial plication with a right thoracotomy had higher incidences of mortality and low cardiac output, and it was stated that this procedure would be effective only to increase lung ventilation. More recently, in 2001, another group (32) compared the two surgical strategies. They showed that the reduction in left atrial size was comparable in both groups. Cox-Maze procedure was used to treat longstanding persistent atrial fibrillation. The aim of this procedure is to divide the macro-reentry circuits and to direct the electrical waves from the sinoatrial node to the atria and atrio-ventricular node. Normal sinus rhythm had been reported to range from $67 \%$ to $98 \%$. (36) Giant left atrium had also been recognized as refractory for regular cardioversion procedures either medically or electrically. (36) This was possibly due to the fibrotic and calcified degeneration of the left atrial myocardium. Yuda et al (36) studied the efficacy of the Maze procedure in restoration of atrial contraction in patients with giant left atrium in comparison to those without giant left atrium. They differentiated between restoration of sinus rhythm on the ECG which reflects the return of the electrical activity and not necessarily the atrial contraction. Return of atrial contraction had been defined in his study as the appearance of A wave on the Doppler mitral inflow pattern by pulsed wave with a peak velocity of at least $10 \mathrm{~cm} / \mathrm{s}$. After one year follow-up, normal sinus rhythm was obtained in 53\% of the giant left atrium in comparison to more than $75 \%$ in non giant left atrium. Atrial contraction was regained in $20 \%$ of patients with giant left atrium in comparison to more than $50 \%$ in non giant left atrium. Another more recent study (37) used the Maze procedure concomitant with mitral valve surgery with or without tricuspid valve surgery and biatrial reduction surgery. The results of this study confirmed the previous one. The bigger the left atrium, the lower is the percent of restoration of sinus rhythm. None of our patients had left atrial placation or Maze procedure. Possibly atrial reduction surgery was not indicated from the start. In view of the absence of compression manifestations, the left atrium dimensions fulfilled the criteria of GLA. Atrial fibrillation rhythm remained and was medically controlled. Anticoagulation prevented thromboembolism. Surgical treatment of giant left atrium with mitral valve disease and atrial fibrillation with complex procedures including mitral valve replacement, atrial reduction surgery with or without Maze procedure is a viable option when indicated. However, we still believe that in cases without intra-cardiac or extracardiac compression, simple mitral valve replacement is sufficient.

\section{Conclusion:-}

Nowadays, giant left atrium is rarely seen and needs a high index of suspicion. It is typically found in patients who have rheumatic mitral valve disease with severe regurgitation. The correct diagnosis of left atrial enlargement is at times not possible by routine CXR alone. It may be misdiagnosed as a mass lesion or pleural or pericardial effusion. Pericardiocentesis, pleurocentesis, or biopsies are dangerous in this setting and other diagnostic modalities are needed.

Progressive LAE might be undetected and well tolerated for a long time, even when it reaches huge proportions.

\section{Reference:-}

1. Rackley CE, Edwards JE, Karp RB: Mitral valve disease. In: The Heart, ed. 7, ed. by Hurst JW. New York: McGraw-Hill, 1990, pp 820-851.

2. Bramlet LA, Edwards JE: Congenital aneurysm of left atrial appendage. Br Heart J 45:97-100, 1985 .

3. Lipkin D, Colli A, Somerville J: Aneurysmal dilatation of left atrial appendage diagnosed by cross sectional echocardiography and surgically removed. Br Heart J 53:69-71, 1985.

4. Pinamonti B, Alberti E, Buttignol G, et al: Echocardiographic diagnosis of congenital left atrail aneurysm. Am Heart J 111:406-409, 1986.

5. Gould LA, Betzu R, Sing Yang DC, et al: Giant left atrium. A case report. Angiology 42:52-54, 1991. 2. Hurst W. Memories of patients with a giant left atrium. Circulation 2001;104:2630-1.

6. Schvartzman PR, White RD. Giant left atrium. Circulation 2001;104:e28-9. 
7. Rimon D, Cohen L, Rosenfeld J. Thrombosed giant left atrium mimicking a mediastinal tumor. Chest 1977;71:406-8.

8. Goldenberg G, Eisen A, Weisenberg N, Amital H. Giant left atrium. IMAJ 2009;11:641.

9. Tung R, DeSanctis R. Giant left atrium. NEJM 2004;351:1437.

10. Fasseas P, Lee-Dorn R, Sokil AB, VanDecker W. Giant left atrium. Texas Heart Inst J 2001;28:158-9.

11. Ates M, Sensoz Y, Abay G, Akcar M. Giant left atrium with rheumatic mitral stenosis. Texas Heart Inst J 2006;33:389-91.

12. Celik T, Bozlar U, Celik M, demirkol S, Iyisoy A, Cingoz F. Giant left atrium in a patient with starr-Edwards caged ball implanted three decades ago. Cardiovasc Revasc Med 2011;12:266-7.

13. Rajakaruna C, Mhandu P, Ghosh-Dastidar M, Desai J. Giant left atrium secondary to severe mixed mitral valve pathology. Eur J Cardio Thoracic Surg 2007;32:932.

14. Kronzon I, Mehta SS. Giant left atrium. Chest 1974;65:677-9.

15. Ananthasubramaniam K, Iyer $G$ and Karthikeyan V. Giant left atrium secondary to tight mitral stenosis leading to acquired Lutembacher syndrome: A case report with emphasis on echocardiography in assessment of Lutembacher syndrome. J Am

16. Soc Echocardiogr 2001;14:1033-5.

17. Funk M, Perez M, Santana O. Asymptomatic giant left atrium. Clin Cardiol 2010;33: e104-5.

18. Agrawal AK, Gupta AK, Gupta VK, Kharb S. Giant left atrium. A rare entity in rheumatic valvular heart disease. J Indian Acad Clin Med 2008;9:227-9.

19. Parrinello G, Torres D, Paterna S, Mezzero M, Di Pasquale P, Trapanese C, et al. Giant left atrium in a woman with mitral

20. prosthetic valve malfunction and history of rheumatic heart disease. Intern Emerg Med 2009;4:435-7.

21. Phua GC, Eng PC, Lim SL, Chua YL. Beyond Ortner's syndrome - Unusual pulmonary complications of the giant left atrium. Ann Acad Med 2005;34:642-5.

22. Brownsberger RJ, Morrelli HF. Hoarseness due to mitral valve prolapse. J Am Geriatr Soc 1988;36:442-3. Yuksel UC, Kursaklioglu H, Celik T. Apical hypertrophic cardiomyopathy with giant left atrium. Arq Bras Cardiol 2007;88:e47.

23. Cheng Z, Fang Q, Liu Y. Cardiac amyloidisis with giant atria. Heart 2010;96:1820.

24. Plaschkes J, Borman J, Merin G, Milwidsky H. Giant left atrium in rheumatic heart disease: A report of 18 cases treated by mitral valve replacement. Ann Surg 1971;174:194-201.

25. Le Roux BT, Gotsman MS. Giant left atrium. Thorax 1970;25:190-8.

26. Minagoe S, Yoshikawa J, Youshida K, Akasaka T, Shakudo M, Maeda K, et al. Obstruction of the inferior vena caval orifice by giant left atrium in patients with mitral stenosis. A Doppler echocardiographic study from the right parasternal approach. Circulation 1992;86:214-25.

27. Castrillo C, Rivas AO, De Berrazueta JR. Giant left atrium mimicking right heart failure. Eur Heart J 2008;29:1056.

28. Tonguç E, Kestelli M, Özsöyler I, Yilik L, Yılmaz A, Özbek C, et al. Limit of indication for placation of giant left atrium. Asian Cardiovasc Thorac Ann 2001;9:24-6.

29. Nigri M, Fernandes JL, Rochitte CE. Giant left atrium and a right sided heart. Heart 2007;93:475.

30. Okyay K, Cengel A, Tavil Y. Images in cardiology. A giant left atrium with two huge thombi without embolic complications. Can J Cardiol 2007;23:1088.

31. Popescu B, Lupescu I, Georgescu S, Ginghina C. Giant left atrium with calcified walls and thrombus in a patient with an old, normally functioning ball-in-cage mitral valve prosthesis. Circulation

32. 2010;122:e579-80.

33. Kutay V, Kirali K, Ekim H, Yakut C. Effect of giant left atrium on thromboembolism after mitral valve replacement. Asian Cardiovasc Thorac Ann 2005;13:107-11.

34. Apostolakis E, Shuhaiber JH. The surgical management of giant left atrium. Eur J Cardiothorac Surg 2008;33:182-90.

35. Tamua Y, Nagasaka S, Abe T, Taniguchi S. Reasonable and effective volume reduction of a giant left atrium associated with mitral valve disease. Ann Thoracic Cardiovasc Surg 2008;14:252-5.

36. Johnson J, Danielson GK, MacVaug H III, Joyner CR. Plication of the giant left atrium at operation for severe mitral regurgitation. Surgery 1967;61:118-21.

37. Yuda A, Nakatini S, Isobe F, Kosakai Y, Miyataki K. Comparative efficacy of the Maze procedure for restoration of atrial contraction in patients with and without giant left atrium associated with mitral valve disease. JACC 1998;31:1097-102. 